\title{
Superstabilization of positive linear electrical circuit by state-feedbacks
}

\author{
T. KACZOREK* \\ Faculty of Electrical Engineering, Białystok Univeristy of Technology, 45D Wiejska St., 15-351 Białystok, Poland
}

\begin{abstract}
The concept of superstability of positive linear electrical circuits is introduced and its properties are characterized. The superstabilization of positive and nonpositive electrical circuits by state-feedbacks is analyzed.
\end{abstract}

Key words: positive, electrical circuit, superstability, state-feedback.

\section{Introduction}

A dynamical system is called positive if its trajectory starting from any nonnegative initial state remains forever in the positive orthant for all nonnegative inputs. An overview of state of the art in positive theory is given in the monographs $[1,2]$. Variety of models having positive behavior can be found in engineering, especially in electrical circuits [3], economics, social sciences, biology and medicine, etc. [1,2].

The positive electrical circuits have been analyzed in [4-11]. The constructability and observability of standard and positive electrical circuits has been addressed in [5], the decoupling zeros in [6] and minimal-phase positive electrical circuits in [7]. A new class of normal positive linear electrical circuits has been introduced in [8]. Positive fractional linear electrical circuits have been investigated in [10] and positive unstable electrical circuits in [11]. Infinite eigenvalue assignment by output-feedback for singular systems has been analyzed in [12]. Zeroing of state variables in descriptor electrical circuits has been addressed in [13]. Controller synthesis for positive linear systems with bounded controls has been investigated in [14]. Stability of continuous-time and discrete-time linear systems with inverse state matrices has been analyzed in [15] and positive stable minimal realization of fractional linear systems in [16]. Superstability and superstabilization of dynamical systems have been considered in [17-19].

In this paper the superstability and superstabilization of positive linear electrical circuits by the state-feedbacks will be addressed.

The paper is organized as follows. In Section 2 the preliminaries concerning the positive linear electrical circuits are recalled. The superstability of positive electrical circuits is introduced in Section 3. The superstabilization of standard and positive electrical circuits by state-feedbacks is analyzed in Section 4 and the superstable positive and nonpositive elec-

*e-mail: kaczorek@isep.pw.edu.pl

Manuscript submitted 2017-05-25, initially accepted for publication 2017-06-19, published in October 2017 trical circuits in Section 5. Concluding remarks are given in Section 6.

The following notation will be used: $\Re$ - the set of real numbers, $\mathfrak{R}^{n \times m}$ - the set of $n \times m$ real matrices, $\mathfrak{R}_{+}^{n \times m}-$ the set of $n \times m$ real matrices with nonnegative entries and $\mathfrak{R}_{+}^{n}=\mathfrak{R}_{+}^{n \times 1}$, $M_{n}$ - the set of $n \times n$ Metzler matrices (real matrices with nonnegative off-diagonal entries), $I_{n}-$ the $n \times n$ identity matrix.

\section{Preliminaries}

Consider the linear continuous-time electrical circuit described by the state equation

$$
\begin{aligned}
& \dot{x}(t)=A x(t)+B u(t), \\
& y(t)=C x(t)+D u(t),
\end{aligned}
$$

where $x(t) \in \mathfrak{R}^{n}, u(t) \in \mathfrak{R}^{m}, y(t) \in \mathfrak{R}^{p}$ are the state, input and output vectors and $A \in \mathfrak{R}^{n \times n}, B \in \mathfrak{R}^{n \times m}, C \in \mathfrak{R}^{p \times n}, D \in \mathfrak{R}^{p \times m}$.

It is well-known [3] that any standard linear electrical circuit composed of resistors, coils, capacitors and voltage (current) sources can be described by the equation (1). Usually as the state variables $x_{1}(t), \ldots, x_{n}(t)$ (the components of the vector $x(t))$ the currents in the coils and voltages on the capacitors are chosen.

Definition 1. [3] The electrical circuit (1) is called (internally) positive if $x(t) \in \mathfrak{R}_{+}^{n}$, for any initial condition $x(0) \in \mathfrak{R}_{+}^{n}$ and every $u(t) \in \mathfrak{R}_{+}^{m}, t \in[0,+\infty)$.

Theorem 1. [3] The electrical circuit (1) is positive if and only if

$$
A \in M_{n}, \quad B \in \mathfrak{R}_{+}^{n \times m}, \quad C \in \mathfrak{R}_{+}^{p \times n}, \quad D \in \mathfrak{R}_{+}^{p \times m} .
$$

Theorem 2. [3] The linear electrical circuit composed of resistors, coils and voltage sources is positive for any values of the 
resistances, inductances and source voltages if the number of coils is less or equal to the number of its linearly independent meshes and the direction of the mesh currents are consistent with the directions of the mesh source voltages.

Theorem 3. [3] The linear electrical circuit composed of resistors, capacitors and voltage sources is not positive for all values of its resistances, capacitances and source voltages if each its branch contains resistor, capacitor and voltage source.

Theorem 4. [3] The $R, L, C, e$ electrical circuits are not positive for any values of its resistances, inductances, capacitances and source voltages if at least one its branch contains coil and capacitor.

Definition 2. The positive electrical circuit (1) for $u(t)=0$ is called asymptotically stable if

$$
\lim _{t \rightarrow \infty} x(t)=0 \text { for all } x(0) \in \mathfrak{R}_{+}^{n} \text {. }
$$

Theorem 5. [3] The positive electrical circuit (1) is asymptotically stable if all coefficients of the characteristic polynomial

$$
\operatorname{det}\left[I_{n} s-A\right]=s^{n}+a_{n-1} s^{n-1}+\ldots+a_{1} s+a_{0}
$$

are positive, i.e. $a_{k}>0$ for $k=0,1, \ldots, n-1$.

\section{Superstability of positive electrical circuits}

Consider the positive electrical circuit for $u(t)=0$ described by

$$
\dot{x}(t)=A x(t)
$$

where $x(t) \in \mathfrak{R}_{+}^{n}$ and

$$
A=\left[\begin{array}{cccc}
-a_{11} & a_{12} & \cdots & a_{1 n} \\
a_{21} & -a_{22} & \cdots & a_{2 n} \\
\vdots & \vdots & \ddots & \vdots \\
a_{n 1} & a_{n 2} & \cdots & -a_{n n}
\end{array}\right] \in M_{n}
$$

Definition 3. The positive electrical circuit (5) (or equivalently the matrix $A$ ) is called superstable if

$$
\sigma(A)=\min _{i}\left(a_{i i}-\sum_{\substack{j=1 \\ j \neq i}}^{n} a_{i j}>0\right) \text { for } i=1,2, \ldots, n .
$$

The set of superstable matrices $A \in M_{n}$ will be denoted by $S_{n}$.

To show the specific properties of the superstable systems (matrices) we shall consider the following examples.
Example 1. Consider the positive system (5) with the matrix

$$
\begin{aligned}
A & =\left[\begin{array}{rr}
-1 & a \\
0 & -2
\end{array}\right] \text { for } a=\{0.5,1,2,4,10\} \\
\text { and } x_{0} & =\left[\begin{array}{l}
x_{10} \\
x_{20}
\end{array}\right]=\left[\begin{array}{l}
1 \\
1
\end{array}\right] .
\end{aligned}
$$

The solution of (5a) with (7) has the form

$$
\begin{aligned}
x(t) & =e^{A t} x_{0}=\left[\begin{array}{cc}
e^{-t} & a\left(e^{-t}-e^{-2 t}\right) \\
0 & e^{-2 t}
\end{array}\right]\left[\begin{array}{l}
1 \\
1
\end{array}\right]= \\
& =\left[\begin{array}{c}
(1+a) e^{-t}-a e^{-2 t} \\
e^{-2 t}
\end{array}\right] .
\end{aligned}
$$

From Fig. 1 it follows that if the coefficient $a$ does not satisfy the condition (6) then the first component $x_{1}(t)$ of $x(t)$ has overshoots over 1 with the amplitudes increasing with the value of $a$.

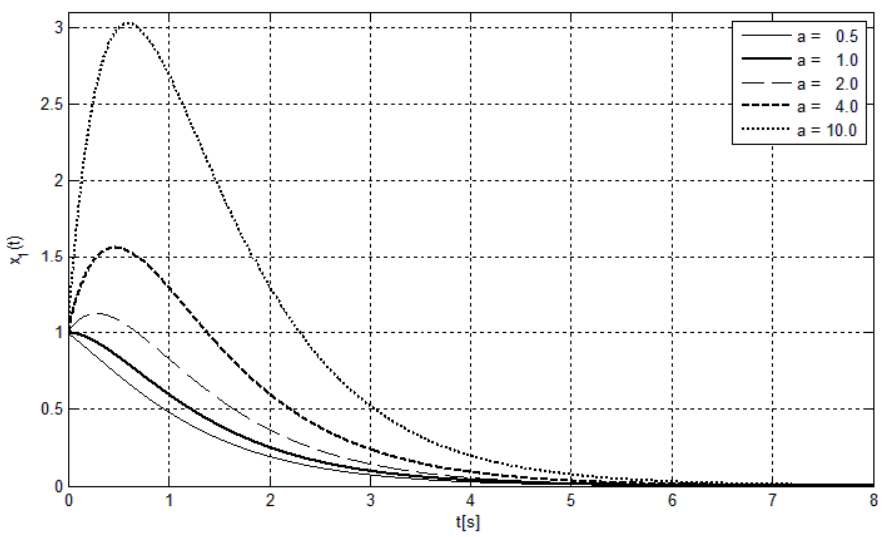

Fig. 1. State variable $x_{1}(t)$ of the system (7) with different values of $a$

Example 2. Consider the positive system (5) with the matrix

$$
\begin{aligned}
A & =\left[\begin{array}{rr}
-1 & a \\
0 & -2
\end{array}\right] \text { for } a=\{0.5,1,2,4,10\} \\
\text { and } x_{0} & =\left[\begin{array}{l}
x_{10} \\
x_{20}
\end{array}\right]=\left[\begin{array}{l}
1 \\
1
\end{array}\right] .
\end{aligned}
$$

The solution of (5a) with (9) has the form

$$
x(t)=e^{A t} x_{0}=\left[\begin{array}{cc}
e^{-t} & a t e^{-t} \\
0 & e^{-t}
\end{array}\right]\left[\begin{array}{l}
1 \\
1
\end{array}\right]=\left[\begin{array}{c}
(1+a t) e^{-t} \\
e^{-t}
\end{array}\right] .
$$

From Fig. 2 it follows that if the coefficient $a$ does not satisfy the condition (6) then the first component $x_{1}(t)$ of $x(t)$ has overshoots over 1 with the amplitudes increasing with the value of 


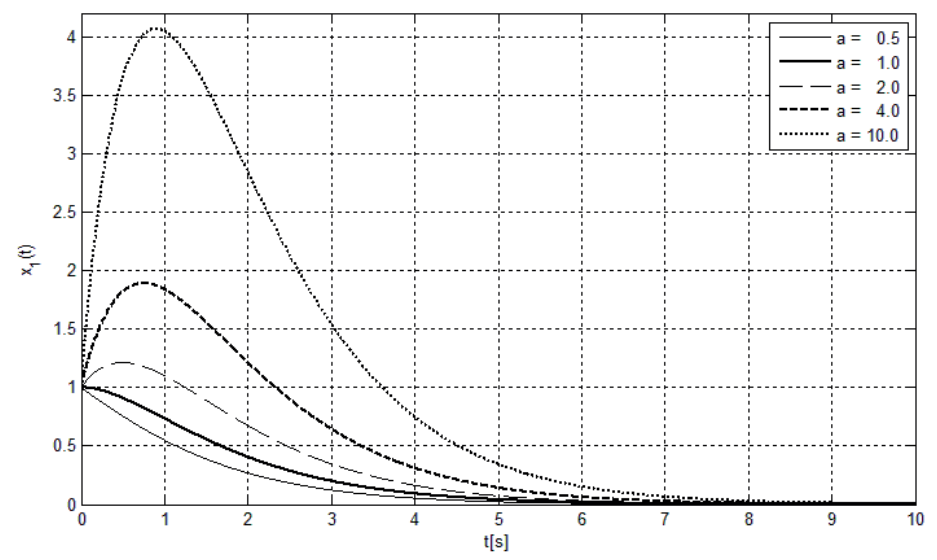

Fig. 2. State variable $x_{1}(t)$ of the system (9) with different values of a

$a$. In many practical (technological) processes such overshoots (peak effects) are not acceptable.

Theorem 6. The state variables $u_{i}(t), i=1, \ldots, n$ (the components of the state vector $x(t))$ of positive superstable linear electrical circuit satisfy the condition

$$
\max _{i} x_{i}(t)=\max _{i} x_{i 0} e^{-\sigma(A) t} \text { for } i=1, \ldots, n,
$$

where $\sigma(A)$ is defined by (6).

Proof. From Gershgorin theorem and (6) it follows that the state variables $u_{i}(t), i=1, \ldots, n$ of positive electrical circuits have not overshoots. See also [17-19].

\section{Superstabilization of standard and positive electrical circuits by state-feedbacks}

Firstly we shall consider nonpositive linear electrical circuits.

Example 3. Consider the electrical circuit shown in Fig. 3 with given resistance $R$, inductance $L$, capacitance $C$ and source voltage $e$.

Using the Kirchhoff's laws we may write the equations

$$
e=L \frac{d i}{d t}+u, \quad i=L \frac{u}{R}+C \frac{d u}{d t},
$$

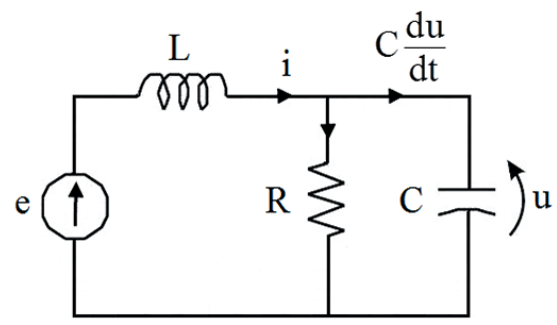

Fig. 3. Electrical circuit of Example 3. which can be written in the form

$$
\frac{d}{d t}\left[\begin{array}{l}
u \\
i
\end{array}\right]=A\left[\begin{array}{l}
u \\
i
\end{array}\right]+B e,
$$

where

$$
A=\left[\begin{array}{cc}
-\frac{1}{R C} & \frac{1}{C} \\
-\frac{1}{L} & 0
\end{array}\right], \quad B=\left[\begin{array}{l}
0 \\
\frac{1}{L}
\end{array}\right] .
$$

From (13b) it follows that the electrical circuit is not positive since $A \notin M_{2}$. Applying the state-feedback

$$
e=\left[\begin{array}{ll}
k_{1} & k_{2}
\end{array}\right]\left[\begin{array}{l}
u \\
i
\end{array}\right]
$$

to the electrical circuit we obtain

$$
\begin{aligned}
A_{C}=A+B K & =\left[\begin{array}{cc}
-\frac{1}{R C} & \frac{1}{C} \\
-\frac{1}{L} & 0
\end{array}\right]+\left[\begin{array}{l}
0 \\
\frac{1}{L}
\end{array}\right]\left[\begin{array}{ll}
k_{1} & k_{2}
\end{array}\right]= \\
& =\left[\begin{array}{cc}
-\frac{1}{R C} & \frac{1}{C} \\
\frac{k_{1}-1}{L} & \frac{k_{2}}{L}
\end{array}\right] .
\end{aligned}
$$

From (15) it follows that for $k_{1} \geq 1$ and $k_{2}<0$ the closedloop system is positive and asymptotically stable but in general case is not superstable since the matrix $A$ does not satisfy the condition (6). The electrical circuit is superstable if and only if $R<1$ and $k_{2}+1>k_{1}$.

Example 4. Consider the electrical circuit shown in Fig. 4 with given resistance $R$, inductance $L$, capacitance $C$ and source voltage $e$. Note that the electrical circuit has been obtained from the electrical circuit in Fig. 3 by interchange the coil with the capacitor.

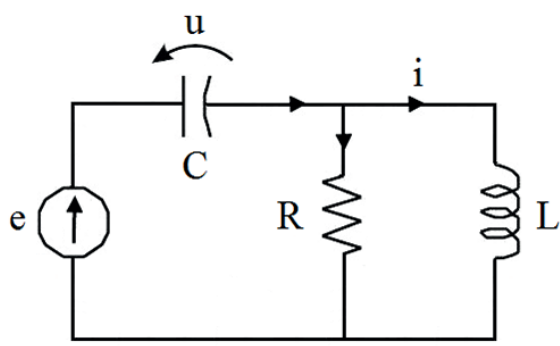

Fig. 4. Electrical circuit of Example 4

Using the Kirchhoff's laws we may write the equations

$$
e=u+L \frac{d i}{d t}, \quad C \frac{d u}{d t}=i+\frac{e-u}{R},
$$

which can be written in the form

$$
\frac{d}{d t}\left[\begin{array}{l}
u \\
i
\end{array}\right]=A\left[\begin{array}{l}
u \\
i
\end{array}\right]+B e,
$$


where

$$
A=\left[\begin{array}{cc}
-\frac{1}{L} & 0 \\
-\frac{1}{R C} & \frac{1}{C}
\end{array}\right], \quad B=\left[\begin{array}{c}
\frac{1}{L} \\
\frac{1}{R C}
\end{array}\right] .
$$

From (17b) it follows that the electrical circuit has the same matrix $A$ as the one in Fig. 3 but different matrix $B$ and it is not positive but asymptotically stable.

Applying the state-feedback

$$
e=\left[\begin{array}{ll}
k_{11} & k_{12} \\
k_{21} & k_{22}
\end{array}\right]\left[\begin{array}{l}
u \\
i
\end{array}\right]
$$

to the electrical circuit we obtain

$$
\begin{aligned}
A_{C}=A+B K & =\left[\begin{array}{cc}
-\frac{1}{L} & 0 \\
-\frac{1}{R C} & \frac{1}{C}
\end{array}\right]+\left[\begin{array}{c}
\frac{1}{L} \\
\frac{1}{R C}
\end{array}\right]\left[\begin{array}{ll}
k_{11} & k_{12} \\
k_{21} & k_{22}
\end{array}\right]= \\
& =\left[\begin{array}{cc}
\frac{1}{L}\left(k_{11}-1\right) & \frac{k_{12}}{L} \\
\frac{k_{21}-1}{R C} & \frac{k_{22}}{R C}
\end{array}\right] .
\end{aligned}
$$

From (19) it follows that the closed-loop electrical circuit is positive if $k_{12}>0, k_{21}>1$ and superstable if and only if $1-k_{11}>k_{12}$ and $1-k_{22}>k_{21}$.

Example 5. Consider the electrical circuit shown in Fig. 5 with given resistance $R$, inductances $L_{1}, L_{2}$ and source voltages $e_{1}, e_{2}$.

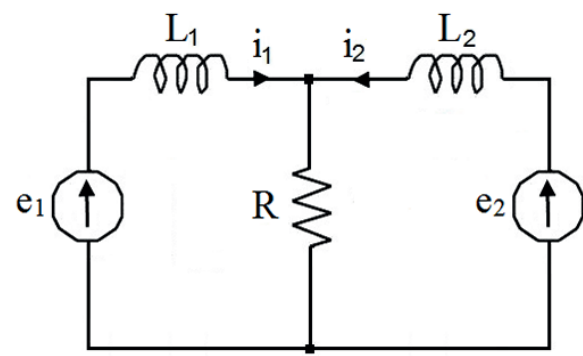

Fig. 5. Electrical circuit of Example 5

Using the Kirchhoff's laws we may write the equations

$$
e_{1}=R\left(i_{1}+i_{2}\right)+L_{1} \frac{d i_{1}}{d t}, \quad e_{2}=R\left(i_{1}+i_{2}\right)+L_{2} \frac{d i_{2}}{d t},
$$

which can be written in the form

$$
\frac{d}{d t}\left[\begin{array}{l}
i_{1} \\
i_{2}
\end{array}\right]=A\left[\begin{array}{l}
i_{1} \\
i_{2}
\end{array}\right]+B\left[\begin{array}{l}
e_{1} \\
e_{2}
\end{array}\right]
$$

where

$$
A=\left[\begin{array}{rr}
-\frac{R}{L_{1}} & -\frac{R}{L_{1}} \\
-\frac{R}{L_{2}} & -\frac{R}{L_{2}}
\end{array}\right], \quad B=\left[\begin{array}{cc}
\frac{1}{L_{1}} & 0 \\
0 & \frac{1}{L_{2}}
\end{array}\right]
$$

From (21b) it follows that the electrical circuit is not positive and unstable since off-diagonal entries of $A$ are negative and $\operatorname{det} A=0$.

Applying the state-feedback

$$
e=\left[\begin{array}{ll}
k_{11} & k_{12} \\
k_{21} & k_{22}
\end{array}\right]\left[\begin{array}{l}
i_{1} \\
i_{2}
\end{array}\right]
$$

to the electrical circuit we obtain

$$
\begin{aligned}
& A_{C}=A+B K=\left[\begin{array}{rr}
-\frac{R}{L_{1}} & -\frac{R}{L_{1}} \\
-\frac{R}{L_{2}} & -\frac{R}{L_{2}}
\end{array}\right]+\left[\begin{array}{cc}
\frac{1}{L_{1}} & 0 \\
0 & \frac{1}{L_{2}}
\end{array}\right]\left[\begin{array}{ll}
k_{11} & k_{12} \\
k_{21} & k_{22}
\end{array}=\right. \\
& =\left[\begin{array}{ll}
\frac{k_{11}-R}{L_{1}} & \frac{k_{12}-R}{L_{1}} \\
\frac{k_{21}-R}{L_{2}} & \frac{k_{22}-R}{L_{2}}
\end{array}\right] \text {. }
\end{aligned}
$$

From (23) it follows that the electrical circuit is positive and superstable if and only if $k_{11}<R, k_{22}<R$ and $2 R>k_{i 1}+k_{i 2}$ for $i=1,2$.

Theorem 4. There exists a gain matrix $K$ such that

$$
A_{C}=A+B K \in M_{n}
$$

only if to each row with at least one off-diagonal negative entry of $A$ the corresponding row of $\mathrm{B}$ is nonzero.

Proof. Let the $i$-th row of $A$ has at least one off-diagonal negative entry and the corresponding $i$-th row of $B$ is zero. Then from (24) it follows that by choice of $K$ we are not able to eliminate the negative entry of $A$.

Theorem 5. By suitable choice of the matrix $K \in \mathfrak{R}^{m \times n}$ it is possible to modify not more than $q=\operatorname{rank} B$ rows of the matrix $A$.

Proof. By Kronecker-Capelli theorem the equation

$$
A_{C}-A=B K
$$

has a solution $K$ if and only if

$$
\operatorname{rank}\left[A_{C}-A, B\right]=\operatorname{rank} B .
$$

Therefore, the maximal number of rows of the matrix $A$ which can be modified by suitable choice of $K$ is $q=\operatorname{rank} B$.

\section{Superstable positive and nonpositive electrical circuits}

In this section nonpositive and positive superstable electrical circuits will be analyzed. 
Example 6. Consider the electrical circuit shown in Fig. 6 with given resistances $R_{1}, R_{2}, R_{3}$, inductances $L_{1}, L_{2}$ and source voltage $e$.

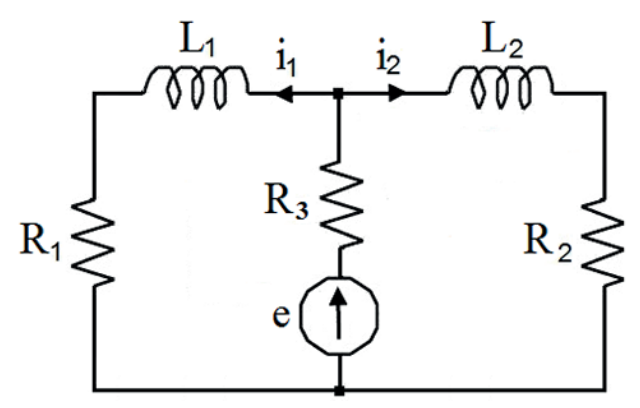

Fig. 6. Electrical circuit of Example 6

Using the Kirchhoff's laws we may write the equations

$$
\begin{aligned}
& e=R_{3}\left(i_{1}+i_{3}\right)+L_{1} \frac{d i_{1}}{d t}+R_{1} i_{1}, \\
& e=R_{3}\left(i_{1}+i_{2}\right)+L_{2} \frac{d i_{2}}{d t}+R_{2} i_{2},
\end{aligned}
$$

which can be written in the form

$$
\frac{d}{d t}\left[\begin{array}{l}
i_{1} \\
i_{2}
\end{array}\right]=A\left[\begin{array}{l}
i_{1} \\
i_{2}
\end{array}\right]+B_{3} e,
$$

where

$$
A=\left[\begin{array}{cc}
-\frac{R_{1}+R_{3}}{L_{1}} & -\frac{R_{3}}{L_{1}} \\
-\frac{R_{3}}{L_{2}} & -\frac{R_{2}+R_{3}}{L_{2}}
\end{array}\right], \quad B=\left[\begin{array}{c}
\frac{1}{L_{1}} \\
\frac{1}{L_{2}}
\end{array}\right] .
$$

From (28b) it follows that the electrical circuit is not positive but superstable since off-diagonal entries of $A$ are negative and the condition (6) is satisfied.

Using the state-feedback

$$
e=\left[\begin{array}{ll}
k_{1} & k_{2}
\end{array}\right]\left[\begin{array}{l}
i_{1} \\
i_{2}
\end{array}\right]
$$

we may modify the dynamics of the electrical circuit since the matrix

$$
\begin{aligned}
A_{C} & =A+B K=\left[\begin{array}{cc}
-\frac{R_{1}+R_{3}}{L_{1}} & -\frac{R_{3}}{L_{1}} \\
-\frac{R_{3}}{L_{2}} & -\frac{R_{2}+R_{3}}{L_{2}}
\end{array}\right]+ \\
& +\left[\begin{array}{c}
\frac{1}{L_{1}} \\
\frac{1}{L_{2}}
\end{array}\right]\left[\begin{array}{ll}
k_{1} & k_{2}
\end{array}\right]=\left[\begin{array}{cc}
\frac{k_{1}-\left(R_{1}+R_{3}\right)}{L_{1}} & \frac{k_{2}-R_{3}}{L_{1}} \\
\frac{k_{1}-R_{3}}{L_{2}} & \frac{k_{2}-\left(R_{2}+R_{3}\right)}{L_{2}}
\end{array}\right]
\end{aligned}
$$

is a superstable Metzler matrix for $k_{i}>R_{3}$ and $R_{i}+2 R_{3}>$ $>k_{1}+k_{2}$ for $i=1,2$.
Example 7. Consider the electrical circuit shown in Fig. 7 with given resistances $R_{1}, R_{2}, R_{3}$ capacitances $C_{1}, C_{2}$ and source voltage $e$.

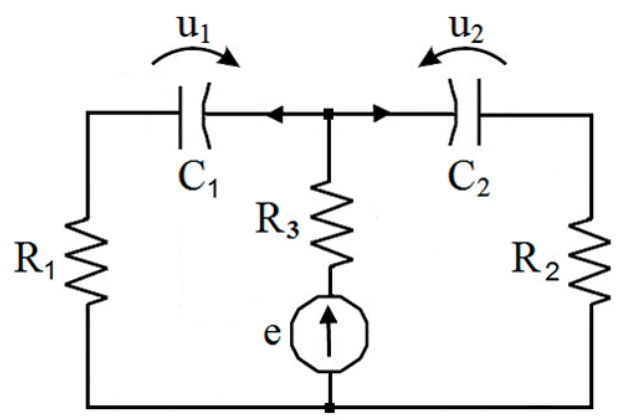

Fig. 7. Electrical circuit of Example 7

Using the Kirchhoff's laws we may write the equations

$$
\begin{aligned}
& e=R_{1} C_{1} \frac{d u_{1}}{d t}+u_{1}+R_{3}\left(C_{1} \frac{d u_{1}}{d t}+C_{2} \frac{d u_{2}}{d t}\right), \\
& e=+R_{3}\left(C_{1} \frac{d u_{1}}{d t}+C_{2} \frac{d u_{2}}{d t}\right)+u_{2}+R_{2} C_{2} \frac{d u_{2}}{d t},
\end{aligned}
$$

which can be written in the form

$$
\begin{aligned}
& {\left[\begin{array}{cc}
\left(R_{1}+R_{3}\right) C_{1} & R_{3} C_{2} \\
R_{3} C_{1} & \left(R_{2}+R_{3}\right) C_{2}
\end{array}\right] \frac{d}{d t}\left[\begin{array}{l}
u_{1} \\
u_{2}
\end{array}\right]=} \\
= & {\left[\begin{array}{rr}
-1 & 0 \\
0 & -1
\end{array}\right]\left[\begin{array}{l}
u_{1} \\
u_{2}
\end{array}\right]+\left[\begin{array}{l}
1 \\
1
\end{array}\right] e . }
\end{aligned}
$$

Premultiplying (32) by the inverse matrix

$$
\begin{gathered}
{\left[\begin{array}{cc}
\left(R_{1}+R_{3}\right) C_{1} & R_{3} C_{2} \\
R_{3} C_{1} & \left(R_{2}+R_{3}\right) C_{2}
\end{array}\right]^{-1}=} \\
=\frac{1}{\Delta}\left[\begin{array}{cc}
\left(R_{2}+R_{3}\right) C_{2} & -R_{3} C_{2} \\
-R_{3} C_{1} & \left(R_{1}+R_{3}\right) C_{1}
\end{array}\right], \\
\Delta=\left[R_{1}\left(R_{2}+R_{3}\right)+R_{2} R_{3}\right] C_{1} C_{2}
\end{gathered}
$$

we obtain

$$
\frac{d}{d t}\left[\begin{array}{l}
u_{1} \\
u_{2}
\end{array}\right]=A\left[\begin{array}{l}
u_{1} \\
u_{2}
\end{array}\right]+B e
$$

where

$$
\begin{aligned}
& A=\frac{1}{\Delta}\left[\begin{array}{cc}
-\left(R_{2}+R_{3}\right) C_{2} & R_{3} C_{2} \\
R_{3} C_{1} & -\left(R_{1}+R_{3}\right) C_{1}
\end{array}\right], \\
& B=\frac{1}{\Delta}\left[\begin{array}{l}
R_{2} C_{2} \\
R_{1} C_{1}
\end{array}\right] .
\end{aligned}
$$

From (34b) it follows that the electrical circuit is positive since $A \subset M_{2}$ and $B$ has positive entries. 
Applying the state-feedback

$$
e=\left[\begin{array}{ll}
k_{1} & k_{2}
\end{array}\right]\left[\begin{array}{l}
u \\
u_{2}
\end{array}\right],
$$

to the electrical circuit we obtain

$$
\begin{aligned}
& A_{C}=A+B K=\frac{1}{\Delta}\left[\begin{array}{cc}
-\left(R_{2}+R_{3}\right) C_{2} & R_{3} C_{2} \\
R_{3} C_{1} & -\left(R_{1}+R_{3}\right) C_{1}
\end{array}\right]+ \\
& +\frac{1}{\Delta}\left[\begin{array}{c}
R_{2} C_{2} \\
R_{1} C_{1}
\end{array}\right]\left[\begin{array}{ll}
k_{1} & k_{2}
\end{array}\right]= \\
& =\frac{1}{\Delta}\left[\begin{array}{cc}
-\left[\left(1-k_{1}\right) R_{2}+R_{3}\right] C_{2} & {\left[R_{3}+k_{2} R_{2}\right] C_{2}} \\
{\left[R_{3}+k_{1} R_{1}\right] C_{1}} & -\left[\left(1-k_{2}\right) R_{1}+R_{3}\right] C_{1}
\end{array}\right] .
\end{aligned}
$$

From (36) it follows that the electrical circuit is positive for $k_{1}>-R_{3} / R_{1}, k_{2}>-R_{3} / R_{2}$ and superstable for $1-k_{1}-k_{2}>0$.

\section{Concluding remarks}

The concept (notion) of superstability of positive linear electrical circuits has been introduced. The specific properties of superstable linear electrical circuits have been characterized. The superstabilization of positive and nonpositive electrical circuits by state-feedbacks has been analyzed.

The considerations can be extended to the descriptor linear electrical circuits.

Acknowledgements. This work was supported by National Science Centre in Poland under work No. 2014/13/B/ST7/03467.

\section{REFERENCES}

[1] L. Farina and S. Rinaldi, Positive Linear Systems. Theory and Applications, J. Wiley, New York, 2000.

[2] T. Kaczorek, Positive $1 D$ and $2 D$ Systems, Springer-Verlag, London, 2002.

[3] T. Kaczorek and K. Rogowski, Fractional Linear Systems and Electrical Circuits, Studies in Systems, Decision and Control, vol. 13, Springer, 2015.
[4] T. Kaczorek, "A class of positive and stable time-varying electrical circuits", Przegląd Elektrotechniczny 91(5), 121-124 (2015) [in Polish].

[5] T. Kaczorek, "Constructability and observability of standard and positive electrical circuits", Przegląd Elektrotechniczny 89(7), 132-136 (2013) [in Polish].

[6] T. Kaczorek, "Decoupling zeros of positive continuous-time linear systems and electrical circuits", Advances in Systems Science. Advances in Intelligent Systems and Computing 240, 1-15 (2014).

[7] T. Kaczorek, "Minimal-phase positive electrical circuits", Przeglad Elektrotechniczny 92(3), 182-189 (2016) [in Polish].

[8] T. Kaczorek, "Normal positive electrical circuits", IET Circuits Theory and Applications 9(5), 691-699 (2015).

[9] T. Kaczorek, "Positive electrical circuits and their reachability", Archives of Electrical Engineering 60(3), 283-301 (2011).

[10] T. Kaczorek, "Positive fractional linear electrical circuits", Proceedings of SPIE, vol. 8903, Bellingham WA, USA, Art. No 3903-35 (2013).

[11] T. Kaczorek, "Positive unstable electrical circuits", Przeglad Elektrotechniczny 88(5a), 187-192 (2012) [in Polish].

[12] T. Kaczorek, "Infinite eigenvalue assignment by output-feedback for singular systems", Int. J. Appl. Math. Comput. Sci. 14(1), 19-23 (2004).

[13] T. Kaczorek, "Zeroing of state variables in descriptor electrical circuits by state-feedbacks", Przeglad Elektrotechniczny 89(10), 200-203 (2013) [in Polish].

[14] M. Ait Rami and F. Tadeo, "Controller synthesis for positive linear systems with bounded controls", IEEE Transactions on Circuits and Systems 54(2), 151-155 (2007).

[15] T. Kaczorek and K. Borawski, "Stability of continuous-time and discrete-time linear systems with inverse state matrices", Measurement Automation Monitoring 62(4), 132-135 (2016).

[16] Ł. Sajewski, "Positive stable minimal realization of fractional discrete-time linear systems", Advances in the Theory and Applications of Non-integer Order Systems, eds. Wojciech Mitkowski, Janusz Kacprzyk, Jerzy Baranowski, Poland in Lecture Notes in Electrical Engineering, vol. 257, 15-30 (2013).

[17] B.T. Polyak and P.S. Shcherbakov, "Superstable linear control systems. I. Analysis", Automation and Remote Control 63(8), 1239-1254 (2002).

[18] B.T. Polyak and P.S. Shcherbakov, "Superstable linear control systems. II. Design", Automation and Remote Control 63(11), 1745-1763 (2002).

[19] Y.V. Talagaev, "Robust analysis and superstabilization of chaotic systems", IEEE Int. Conf. on Control Applications, Juan Les Antibes, 1431-1436 (2014). 